\title{
Quelques éléments du discours dit " islamiste » : cas du Parti de la Justice et du Développement du Maroc
}

\author{
Sara Mejdoubi ${ }^{1}$ \\ Université Internationale de Rabat
}

\begin{abstract}
Résumé. Notre travail vise à définir les multiples manifestations du discours islamiste du Parti de la Justice et du Développement (PJD). En se basant sur les travaux de $\mathrm{D}$. Maingueneau, notre étude cherche à distinguer les manifestations langagières à travers des unités topiques, soumises à un prédécoupage social, qui relèvent de genres et de types institués ; c'est-àdire qui ne se réalisent qu'à travers des lieux institutionnels. Nous distinguons donc les unités domaniales du discours qui se déploient à travers trois catégories, à savoir la sphère d'activité, le champ discursif et les lieux d'activité. Outre ces unités prédécoupées, nous abordons l'activité discursive islamiste grâce à la notion de formation discursive, qui permet d'inscrire le discours PJDiste dans une idéologie nourrie de divers positionnements.
\end{abstract}

\begin{abstract}
Some elements of the alleged « islamist» discourse : Case of the Moroccan Justice and Development party. We aim to define the multiple embodiments of the Islamist speech of the Moroccan Justice and Development Party (PJD). Based on the work of D. Maingueneau, our study identifies the language embodiments through topical units, subject to various social mediums of communication, which reveal the established genres and types; that are only achieved through institutional mediums. We segment the domain units of the speech which are unfolded through three elements, namely the sphere of activity, the speechful field and the places of activity. In addition to the segmented units, we approach the Islamist speechful activities thanks to the notion of speechful formation, which makes it possible to classify the "PJDistic" speech as an ideology inspired from various positions.
\end{abstract}

\begin{abstract}
Analyser l'islamisme, comme activité discursive, revient à aborder ses conditions de productions à travers les différentes expressions langagières. La parole islamiste se manifeste à travers un lieu institutionnel qui l'autorise et un positionnement idéologique qui la caractérise et permet son identification. Cependant, lorsque nous entreprenons de cerner la question du discours islamiste contemporain, nous nous heurtons d'emblée à la question de l'idéologie sous-jacente qui le rend visible et saisissable. Des chercheurs tels que A.-C. Larroque, M. El Ahmadi, O. Carré, J.-F. Clément, O. Roy, F. Burgat, ainsi que A.
\end{abstract}


Lamchichi, pour ne citer que ceux-là, ont déjà constaté que l'idéologie islamiste est traversée par une forte variabilité. Son évolution, dans tel ou tel contexte, dépend de considérations historiques, politiques, culturelles, sociales et religieuses. Une cartographie qui pourrait représenter, avec exactitude, les formes de l'idéologie islamiste, à travers le monde, serait laborieuse : cela reviendrait à l'ajuster d'une façon constante. Il n'en est pas autrement quand il s'agit de définir le discours qui lui correspond. Pour autant et malgré les différences des modes opératoires dans les pays qui hébergent les mouvements et les organisations islamistes, les valeurs, les orientations, les représentations et les symboles de l'organisation égyptienne des Frères musulmans, constituent l'ossature idéologique de l'islamisme politique du XXe siècle (Larroque 2018 : 62) et, par conséquent, de son discours.

Nous envisageons, à travers cet article, d'identifier les particularités, les manifestations et les caractéristiques fondamentales du discours du parti politique marocain, intitulé Parti de la Justice et du Développement (PJD). Le discours islamiste est marqué par un positionnement idéologique déterminé. Cependant, en gommant les accessoires contextuels, il s'agit clairement d'une position qui définit le monde selon des principes et des représentations qui ne s'identifient que par opposition à un autre mode de définition. L'islamisme propose une vision manichéenne du monde où le bien et le mal ne s'entremêlent pas et sont même en confrontation. Dans ce sens, A. Dialmy explique que l'islamiste «ne se pose qu'en s'opposant à des ennemis, à des autres. Le rapport qui relie le Moi islamiste aux autres, à l'Autre, est un rapport essentiellement conflictuel » (Dialmy 2000). Au-delà de cette dichotomie, le message islamiste, sommaire et schématique, se distingue par son désir d'universalité. Son objectif est de guider toute l'humanité sans exception aucune (Carré 1984 : 123).

Nous n'allons pas nous aventurer dans des définitions aléatoires de l'islamisme. Nous partirons du postulat selon lequel l'islamisme est un mouvement, un groupement d'hommes et de femmes qui s'engagent dans la lutte politique en s'appuyant sur le texte coranique, la Sira du prophète de l'islam, la conduite de ses compagnons et sur l'importance des exégètes qui ont eu, tout au long des siècles, une influence considérable sur les musulmans. Comme on le sait, l'islam offre, à cet égard, aux musulmans une réelle vision politique de la société : une gouvernance selon des lois, des pratiques, des processus et des institutions, vision qui serait intemporelle. Néanmoins, l'idée de saisir le fait politique, en considérant que les lois sont immuables, présente une difficulté pour l'analyste. Le discours est envisagé, à son tour, comme intemporel transcendant le temps et l'espace, donc le contexte. Pour mieux accompagner son désir d'universalité, il se veut un discours diffus et difficile à saisir.

Dans cette optique, le discours politique dit « islamiste » représente un ensemble d'unités topiques et non-topiques qui ne serait saisi que dans un processus d'influence qui accompagne son désir d'universalité. Nous estimons que c'est pour cette raison que les manifestations de ce discours ne pourraient se limiter uniquement à 'ce qui est dit', à un ensemble d'énoncés produits dans le cadre d'une formation politique (association ou parti politique). Le message politique islamiste émerge, sous la forme d'unités qui obéissent à un prédécoupage social et notamment sous la forme d'un ensemble plus vaste qui emprunte à des formations socio-discursives plus larges. En effet, J.-M. Adam et P. Charaudeau ont opté pour une démarche qui saisit le discours dans le cadre d'une dimension à la fois sociale et textuelle. D. Maingueneau propose d'approfondir l'analyse à travers la distinction entre deux types d'unités de discours. Selon lui, les types et les genres de discours s'inscrivent dans ce qu'il appelle les unités topiques et non-topiques. L'analyste explique que le discours se manifeste à la fois à travers des espaces limités, qui obéissent à un découpage particulier, et d'autres qui transcendent ces espaces. Décrire le discours islamiste au Maroc comme genre et type de discours est une manière de saisir les différentes manifestations dans un cadre communicationnel bien précis. L’École française d'analyse de 
discours nous offre un cadre théorique et des modèles d'analyse assez complets qui nous permettront d'arriver à notre objectif : définir le discours islamiste. En se basant sur la démarche de Dominique Maingueneau, nous tenterons donc de penser le discours islamiste dans le cadre des unités topiques et des unités non-topiques qui s'inscrivent dans un interdiscours. Toutefois, ce travail se limitera à l'étude des unités domaniales et à cerner la notion de formation discursive en raison de l'intérêt qu'elles présentent dans notre objectif de définir le discours islamiste du PJD.

\section{Les unités topiques du discours islamiste}

Les unités topiques, selon D. Maingueneau, sont de deux sortes : domaniales et transverses (Maingueneau 2009 : 127). La première catégorie est intimement liée au type de discours consacré à un secteur d'activité de la société notamment par les pratiques verbales (Maingueneau 2005 : 64-75). Il s'agit là d'un champ de pratiques, soumis à un cadre référentiel déterminé, concourant à un objectif commun. Ce cadre référentiel peut être distinct, quand il fait référence à des groupements par champ idéologique, politique, administratif ou religieux ; comme il peut constituer un type de discours hybride, issu d'un arrangement implicite entre deux types de discours, voire plus. Cette dernière précision constitue notre postulat sur le discours islamiste comme type de discours. Outre le type de discours, les unités domaniales sont aussi en relation avec la notion de genre de discours qui est soumise à un ensemble de conditions de production. D. Maingueneau définit le genre comme " une institution de la parole » et un " dispositif de communication sociohistoriquement déterminé » (Maingueneau 2014 : 64). L'analyste peut procéder à trois types de groupement du discours comme genre (Ibid. : 65-67). S'agissant du discours politique, comme type de discours, ses genres peuvent être regroupés selon :

i) une sphère d'activité, relative au domaine de la politique. Cette sphère n'est pas un espace homogène dans le sens où les genres peuvent constituer un "noyau », tels les discours qui rassemblent l'orateur politique et son auditoire ; comme ils peuvent constituer une « périphérie » qui concerne tout ce qui gravite autour de l'activité politique : réunions entre les membres d'un même parti ou éditoriaux d'une presse partisane ;

ii) des champs discursifs où s'affrontent divers positionnements, dans un même espace, soumis à une logique de confrontation et de rapports de force ;

iii) les lieux d'activité qui concernent, selon Maingueneau, le réseau des genres qui se produisent dans des lieux institutionnels, tels que les genres qui circulent au sein du parlement.

Les unités topiques peuvent également être transverses (Maingueneau 2005 : 64-75). Ces unités, indépendantes de l'appareil institutionnel, traversent les espaces prédécoupés et inscrivent les pratiques discursives dans des frontières selon trois types de registres : (i) le registre défini à partir des critères linguistiques (que l'on peut établir à partir de la théorie de l'énonciation) ; (ii) le registre qui se base sur des critères fonctionnels (selon la fonction que le discours accompli) ; (iii) le registre qui assemble les critères linguistiques et fonctionnels et qui relève de l'ordre du communicationnel (selon l'objectif - didactique, comique, propagandiste - auquel souhaiterait aboutir le discours).

\subsection{Les unités domaniales du discours islamiste}

Depuis son arrivée au pouvoir, en 2011, le PJD dispose d'un arsenal communicationnel qui assure une diffusion structurée et institutionnalisée de sa parole ${ }^{i}$. Cette parole se réalise dans des lieux tels que le parlement, les ministères, le siège physique du parti, la presse qui 
lui est proche, le site internet officiel et les différentes pages personnels dont disposent ses leaders. Cependant, le groupement par genre dépend des dispositifs de communication, associés à un lieu et à un moment déterminé, qui rendent possible la parole politique (Maingueneau2010 : 85-90). Il est nécessaire donc, d'apporter quelques précisons élémentaires au sujet de la langue ${ }^{\mathrm{ii}}$ - ou des langues - de l'énonciation. Il s'agit de l'arabe standard et de l'arabe dit 'marocain'. Chacun dispose d'un arsenal symbolique qui lui assure pouvoir et légitimité. La langue arabe dispose d'une double légitimité : une légitimité religieuse et culturelle qu'elle tire de son statut de langue du Coran et langue de la culture arabo-islamique (toutes les prières se déroulent en langue arabe) et une légitimité politique et nationale qu'elle tire de son statut de « langue officielle de l'état ».

Symboliquement, cette langue a une sacralité quasi-divine aux yeux des marocains. En d'autres termes, elle permet et facilite la reconnaissance et l'appartenance des marocains à une entité plus large, celle de la "Oumma islamiya » (la communauté musulmane). Une entité possédant une identité commune qui transcende les particularismes ethniques, géographiques, historiques, socioculturels et linguistiques.

Cependant, le parler marocain, communément appelé darija, arabe dialectal ou arabe parlé, dispose à son tour d'un pouvoir réel et symbolique très puissant : il est la langue maternelle d'une grande partie de la population et la «langue véhiculaire entre arabophones et berbérophones " (Ziamari-De Ruiter 2015 : 441-462). La darija, lieu de la matérialisation de la doxa, est devenue la lingua franca des hommes et des femmes politiques au Maroc. Plus que l'arabe classique, plus que le berbère, plus que tout autre langue, c'est la darija qui leur permet de se rapprocher du système de pensée des marocains et de se faire comprendre de tous les citoyens.

Ces langues, en usage dans des lieux et des moments légitimes, contribuent à l'établissement et au maintien de ce que P. Charaudeau a nommé un "contrat de parole » (Charaudeau 1983 : 50). Car, en dehors des lois qui gouvernent le discours ${ }^{\mathrm{iii}}$, P. Charaudeau précise que le discours politique, comme acte de parole, est un acte mis en scène nécessitant un contrat, c'est-à-dire une proposition que le sujet communiquant fait à un sujet interprétant. Celle-ci est jonchée de «filtres constructeurs de sens » (Ibid.) qui permettent l'établissement, entre les deux sujets, d'un accord fonctionnant sur la base d'une construction d'une image que le premier renvoie au second (Ibid. : 51-52). Cette notion de contrat comprend un ordre énonciatif particulier qui permet de construire le rapport entre les protagonistes, dans un cadre donné, en définissant leurs statuts respectifs (Ibid. : 59). Nous nous intéressons ici à la scène d'énonciation - une notion qui se rapporte aux textes et non aux phrases - qui se compose d'une scène englobante, relative au type du discours, et d'une scène générique qui a trait au genre de discours (Maingueneau 2012 :78). S'ajoute, aux deux scènes précitées, la scénographie qui est la plus à même de présenter des indications permettant ainsi au destinataire d'identifier le discours en tant que message politique.

Pour reprendre Maingueneau, la scénographie, par l'énonciation, impose un dispositif de parole qui permet de toucher le public en facilitant la légitimation, par le destinataire interprétant, du message du sujet communiquant (Ibid. : 80).

\subsubsection{La sphère d'activité}

Si l'on se rapporte au découpage opéré par D. Maingueneau, les unités domaniales du discours islamiste se réalisent dans un premier temps à travers une sphère d'activité qui dispose d'un noyau et d'une périphérie. Depuis 2011, en période électorale ou en dehors des élections, les leaders du PJD, principalement les chefs de gouvernement, Saâdeddine El Othmani et avant lui Abdelilah Benkirane, s'adressent à l'auditoire de différentes façons. D'abord, à travers des meetings, dans différentes villes du Maroc, rassemblant un auditoire de sympathisants conquis par l'idéologie PJDiste. Le meeting, comme scène englobante, 
représente un classique en matière du discours politique et le genre fétiche du parti islamiste, son arme de séduction massive. C'est le moment où, l'orateur islamiste, rappelle les éléments clés de son projet et le consolide en s'opposant à des adversaires. Le sujet communiquant et le destinataire interprétant sont liés dans le cadre d'un espace-temps bien précis. Dans ces conditions, le message est identifié et légitimé par l'auditoire. Comme scène générique, le meeting est un lieu où une figure emblématique du parti prend la parole, en énonçant une parole publique. Cette parole est énoncée généralement en darija pour A. Benkirane, ce qui lui confère un impact immédiat sur l'auditoire. Un autre aspect significatif, de la scène générique, réside dans le fait que le contrat - ajouté au pouvoir du parler marocain - qui lie les partenaires de la scène d'énonciation est préalablement admis, les propos sont reconnus et ovationnés. A ce cadre scénique, qui se compose de la scène englobante et de la scène générique, s'ajoute la scénographie qui accentue le pouvoir de la mise en scène et celui des représentations langagières. Au Maroc, ce rituel n'est pas ancien. Ce n'est pas un meeting à l'américaine, avec ses stars venues chanter et soutenir le candidat avant son discours, ce qui joue un rôle important dans la scénographie. Non, c'est plus simple et infiniment moins couteux : les invités sont installés au premier rang dans les meetings du PJD, dans lesquels Benkirane a l'habitude de se présenter, à travers son discours, comme le protecteur du peuple, et qui se dresse, selon sa terminologie, devant " les démons et les crocodiles » qui tendraient à déstabiliser la foi et la nation. Les anecdotes qui jonchent son discours sont nombreuses; chacune renvoie à des situations où l'auditoire s'y reconnaît facilement. Il évoque constamment des histoires de son quotidien et de son parcours pour illustrer son combat pour le peuple. Généralement, le meeting se caractérise par une scénographie diffuse qui fait appel à des situations éparses et sans lien apparent.

Outre les meetings politiques, les conférences dans les universités, comme sphère d'activité, représentent un genre particulier pour le discours islamiste. Sa scène englobante tient du type politique et du type didactique à la fois. Politique : le positionnement rappelle le référentiel et l'idéologie islamiste du parti ; didactique : le discours est prononcé devant un parterre d'étudiants assez hétérogène. Le cadre est un espace institutionnel propre à l'enseignement supérieur réglé par un temps de parole limité et imposé par les organisateurs. L'intérêt que représente la conférence, comme genre de discours, c'est la possibilité d'interaction qui suit la fin de la prise de parole de l'orateur. La particularité de la scène générique de la conférence, comme lieu du discours politique, est relative au contrat qui, contrairement au meeting politique, implique un auditoire qui n'est pas le destinataire habituel du sujet communiquant. L'auditoire - composé d'étudiants et d'enseignants - et l'orateur, n'appartenant pas forcément aux mêmes pratiques sociales, ne partagent pas les mêmes représentations langagières qui se rattachent d'un côté au discours didactique et de l'autre côté au discours islamiste. Le débat qui s'ensuit ne fait que confirmer la nature de la relation qui lie les deux parties et met sur le même pied d'égalité les rôles liés au contrat auquel on s'attend entre l'homme politique et son auditoire : le droit questionner et le devoir de répondre. Par ailleurs, A. Benkirane, comme dans ses meetings, ne modifie pas beaucoup ses scénographies, lors de ses conférences, lesquelles sont reconnues aisément par l'auditoire. La conférence représente une périphérie de la sphère d'activité, car le cadre est différent en général de celui du discours politique dans les meetings. Ceci n'empêche pas les leaders du PJD de rappeler leur " référentiel $^{\text {iv }}$ » et leur attachement aux préceptes de l'islam, ce qui confère au discours un versant religieux. D'ailleurs, il n'est pas rare que Benkirane, par des versets coraniques et des Hadiths du prophète, donne à ses paroles le ton des discours religieux.

C'est principalement à travers ces deux genres - le meeting et la conférence - que la force démonstrative du PJD est la plus remarquable. Observons que depuis 2014, une bonne partie de l'arsenal communicatif du PJD se déploie sur Internet. 


\subsubsection{Le champ discursif}

Saisir le discours islamiste au Maroc, comme champ discursif, revient à considérer l'ensemble des positionnements qui composent cette mouvance. En effet, la mouvance islamiste marocaine est composée de plusieurs sous-champs, qui disposent à leur tour de positionnements assez variés. Nous allons considérer, dans le cadre de cette étude, ce que Maingueneau appelle un espace discursif" (Maingueneau 2009 : 23-24) car nous ne mettrons l'accent que sur les positionnements discursifs qui s'affrontent à l'intérieur du PJD comme sous-champ de la mouvance islamiste marocaine.

Le PJD s'est établi sur les décombres du mouvement Ac-chabiba al Islamiya qui, selon A. Dialmy, se démarquait déjà par ses deux principales positions : l'une clandestine et violente, l'autre légal et apostolique (Dialmy 2000). La première a fini par s'essouffler grâce notamment à Abdelilah Benkirane qui a voulu sortir de la clandestinité et intégrer légalement l'échiquier politique. Ceci s'est réalisé à travers la création de la Jamâ'a islâmiyya, une association dont l'objectif est la prédication religieuse (Belal 2009). Malgré l'image d'un parti uni, que depuis 2011 les membres n'arrêtent pas de promouvoir, nous relevons de nombreuses positions qui s'entrecroisent en son sein. Celles-ci s'articulent principalement autour des libertés individuelles et certaines " affaires » qui ont touché quelques-uns de ses ministres et député-e-s. L'« affaire » qui a marqué l'opinion publique récemment, et qui a divisé la «famille » du PJD, est celle de la parlementaire Amina Maelainine, critiquée pour ses photos, devant le Moulin rouge à Paris, sans voile. La députée a été attaquée notamment par Mustapha Ramid, ministre d'État chargé des Droits de l'Homme, qui n'a pas hésité de brandir la traduction de la députée devant le « conseil de discipline » du parti et de « sanctions ${ }^{\mathrm{vi}}$ à son encontre.

Certes, d'autres positions méritent d'être citées, mais nous souhaitons mettre l'accent sur des rapports de force assez évidents qui, émanant du Parti de la Justice et du Développement, finissent par diviser ses rangs, ses partisans et l'opinion publique.

\subsubsection{Les lieux d'activité}

Nous allons procéder, à présent, à l'étude du réseau de genres qui sont en usage dans quatre lieux institutionnels, à savoir le siège du parti, le parlement, les médias, Internet avec ses réseaux sociaux.

\subsubsection{Les genres relatifs au siège du partivii}

Il s'agit, dans un premier temps de textes fondateurs concernant la gestion des affaires internes du parti et sa vision stratégique, à savoir les statuts, les textes de loi relatifs à l'organisation des affaires du parlement, le programme politique, la synthèse du bilan du groupe parlementaire et les textes reprenant les objets et les objectifs des congrès nationaux organisés par le parti. Ces genres, qui constituent des textes fondamentaux pour le fonctionnement du parti, relatent principalement son idéologie et sa vision pour le Maroc. Ils sont souvent signés par le secrétaire général du parti. L'autre catégorie de genres, qui circule au sein du parti, concerne les multiples déclarations relatives à ce que ses membres appellent le « dialogue interne ». Ce dernier s'articule généralement autour des projets d'amélioration et de consolidation des acquis ${ }^{\text {viii }}$. S'ajoute, à ce genre, celui relatif aux différentes déclarations du secrétariat général - abordant les grands sujets discutés lors des réunions internes - et celui qui concerne les déclarations des partis de la majorité.

\subsubsection{Les genres relatifs au parlement}


Le parlement marocain est bicaméral, composé de deux chambres : la chambre des représentants et la chambre des conseillers. La langue de travail est l'arabe et accessoirement le berbère. Il arrive aux députés, conseillers et membres du gouvernement de s'exprimer en darija. Les débats au sein du parlement sont marqués par une «quête de légitimité » (Le Bart 1998 : 79) permanente qui pousse les multiples orateurs à user de plusieurs stratégies discursives afin de marquer le bien-fondé de leurs positionnements politiques. La scène d'énonciation, qui renforce cette quête de légitimité, est marquée par une scène générique qui renvoie constamment à des indications d'espace, de temps et de fonctions des personnes qui interagissent. Ainsi, le Parlement, par exemple, peut être désigné de plusieurs façons, telles que : « cet endroit », " ces murs », " cette estrade » ou « ce lieu de démocratie $»^{\text {ix }}$. En prenant la parole au sein du parlement, A. Benkirane rappelle sa posture, de chef du gouvernement marocain, à travers plusieurs taxèmes (Kerbrat-Orecchioni 1992 : 75). Ainsi, son intensité articulatoire pour monopoliser la parole, l'alternance codique qu'il effectue entre l'arabe et le parler marocain, le fait d'interpeller son auditoire avec des questions, le rappelle de sa fonction et le fait d'avoir été « élu par le peuple », sont autant de signes qui rappellent sa légitimité et la hiérarchie qui existe entre lui et son auditoire.

\subsubsection{Les genres relatifs aux médias}

Le porte-voix du projet politique et sociétal du Parti islamiste est un organe de presse arabophone fondé en $1999^{\mathrm{x}}$. Attajdid $^{x i}$, à la fois lié au Mouvement Unicité et Réforme ${ }^{\mathrm{xii}}$ l'aile idéologique du PJD - et au PJD, est un journal hebdomadaire. Ce support d'expression des leaders PJDistes ${ }^{\text {xiii }}$ est constitué de plusieurs rubriques : « politique », " régions et provinces ", " économie et développement », « arabe et monde », " famille et société » et « vie et religion ». Les genres que l'on y trouve sont assez variés. Si l'on se rapporte à la polarisation effectuée par J.-M. Adam (Adam 1997), laquelle repose sur l'approche énonciative, nous distinguons :

i) le pôle distance-information : reportages, interviews journalistiques, biographies journalistiques, brèves, hommages, portraits, etc., présentant des articles qui ne portent pas beaucoup les traces de leurs auteurs ; celles-ci sont bien implicites ;

ii) le pôle implication-commentaire : le «statement», l'éditorial, le billet, la critique, etc., affichant explicitement l'opinion de leurs auteurs.

De toute évidence, le genre où l'on constate des positions tranchantes et sans équivoque est l'éditorial de Attajdid. Une étude de cet l'éditorial (Mejdoubi 2013 : 34-35) montre que le plan d'énonciation du type discours (Benveniste $1966: 259-260)$ est caractéristique de ce genre, car nous notons des prises de positions et des jugements (les adverbes), une réflexion (le conditionnel et les modalisateurs), des comparaisons ainsi que la présence d'un énonciateur qui se manifeste principalement à travers le " nous ». Par ailleurs, ce qui caractérise l'éditorial, comme mode d'expression, c'est qu'il appartient à la fois au type de discours politique (prise de position idéologique manifeste) et au type de discours journalistique (qui répond à des exigences qui dépendent de la situation de communication) avec un seul objectif : celui d'influencer et d'orienter. Le 30 mars 2017, la publication de ce journal s'est définitivement arrêtée, réorientant l'attention des leaders du parti vers le numérique. Malgré cet arrêt, le parti s'est lancé dans la publication d'un magazine, baptisé « Justice et Développement », censé publier des statistiques, des « données officiels » et de « mettre fin aux rumeurs $»^{\text {xiv }}$.

Outre ces genres médiatiques écrits, les leaders du PJD s'expriment sur les chaînes de télévision nationales ( $S N R T$ et $2 M$ ) dans le cadre de débats politiques, et internationales dans le cadre d'interviews ${ }^{\mathrm{xv}}$. A ce propos, nous mentionnons le passage de Abdelilah 
Benkirane qui a été l'invité du journaliste Ahmad Mansour, de la chaîne Al Jazeera, dans l'émission Bila Houdoud - que l'on pourrait traduire "sans limites » - durant laquelle, le chef du gouvernement a été questionné, en langue arabe, sur le bilan de son gouvernement, la nature des oppositions qu'il rencontre et les différents projets du parti.

\subsubsection{Les genres diffusés sur Internet et les réseaux sociaux}

Internet est sans conteste le lieu où les manifestations du discours du PJD sont les plus diversifiées. Les pratiques discursives, qui s'y trouvent, interpellent l'analyste quant à la quantité, la variété des sujets abordés et leurs modes de mise en ligne. Ainsi, son site officiel $^{\mathrm{xvi}}$ est actualisé en permanence et offre à l'internaute une multitude de rubriques, relevant du genre journalistique, sur des sujets divers : politiques, économiques, sociétaux et culturels. Le site propose aussi des « lettres d'information », une " photothèque » et un espace « forum ». Il permet d'accéder à une « web radio » ${ }^{\text {xvii }}$ - qui diffuse ses programmes en direct - et à une "web tv » ${ }^{\text {xiii }}$, en langue arabe, opérationnelle depuis 2017. Cette dernière offre, dans une certaine mesure, à l'internaute les mêmes programmes et les mêmes genres qu'une chaîne parlementaire classique, à la différence près que l'objet de l'information gravite essentiellement autour des activités et des manifestations du chef du gouvernement, des ministres et des leaders du parti. Grâce à cette chaîne partisane unique au Maroc, le PJD a pris une longueur d'avance sur les autres partis politiques marocains qui peinent à trouver une place sur la toile.

A cela s'ajoute la page officielle du parti sur le réseau social Facebook ${ }^{\text {xix }}$ - ainsi que les pages officielles des cadres et responsables du parti - où l'on trouve des indications générales sur le parti et ses liens officiels avec d'autres réseaux sociaux. Une chaîne officielle du parti sur la plateforme Youtube $e^{x x}$ reprend, à son tour, la diffusion des entretiens, des reportages et des informations publiés sur la chaîne en ligne. La plateforme Twitter accueille aussi une page officielle, depuis novembre 2011, à travers laquelle le PJD diffuse, en continu, des déclarations du chef de du gouvernement, des articles de presse abordant les activités et les déclarations des ministres ou député-e-s du parti.

Cette présence massive sur Internet est propre au PJD. Aucune autre formation politique marocaine ne peut se vanter d'offrir au citoyen-internaute un champ d'information aussi varié que celui du PJD. Internet est devenu, pour le parti islamiste, un lieu institutionnel, d'influence massive et de mobilisation que les élections législatives de 2016 ont largement confirmées.

\section{Les unités non-topiques du discours islamiste}

Contrairement aux unités topiques, soumises à un prédécoupage social, les unités nontopiques représentent des espaces qui dépassent ces frontières. Elles sont constituées par le chercheur et sont profondément inscrites dans l'histoire. D. Maingueneau en distingue deux catégories : les parcours et les formations discursives. Les parcours permettent « d'explorer une dispersion et une instabilité et non de rapporter une séquence verbale à une source énonciative » (Maingueneau 2011). Le but est de décrire les modifications d'une formule dans différents textes ou dans différents contextes sans les ramener à un positionnement défini. Nous n'allons pas pouvoir aborder les parcours, dans le cadre de ce travail, car ils nécessitent des analyses précises et détaillées qui font appel, par ailleurs, à la lexicométrie. Nous allons nous concentrer sur la notion de formation discursive qui est la plus à même de rendre compte des énoncés qui transcendent les unités topiques. Cette notion, détaillée dans le point qui suit, a connu une genèse loin du cadre de l'analyse du discours, mais a le mérite de rendre compte de l'étendu du discours islamiste. 


\subsection{La formation discursive : de M. Foucault à D. Maingueneau}

M. Foucault envisage le concept de formation discursive comme un ensemble de structures hybrides dont les contours transcendent les unités préalablement déterminées par les contraintes liées aux genres et aux types de discours. Cet ensemble dit "invisible » pourrait, selon Foucault, expliquer un certain nombre de phénomènes (Foucault 1969 : 4244). Dans le même sillage, M. Pêcheux reprend le concept, au profit de l'analyse de discours, en l'associant à la philosophie althussérienne qui décrivait les confrontations sociales en termes de "formation social » et de " formation idéologique » (Pêcheux et al. 1971). Ces deux tendances sont jonchées de difficultés pour l'analyste qui se positionne dans une problématique discursive et sociale à la fois. D'une part, la première tendance demeure assez instable quant à la valeur de la notion de « formation discursive » qui varie entre " un système de pensées », " règles ", " système " et " dispersion » (Maingueneau 2011). La seconde tendance, qui se place dans le champ de la lutte politique, met plutôt l'accent sur la « position » que sur celle du " genre » dont l'importance est déterminante quand on se positionne dans une problématique de l'analyse de discours (Ibid.).

Si l'on s'inscrit dans la démarche de M. Foucault, l'islamisme pourrait être représenté comme le comportement langagier d'un ensemble d'individus obéissant à des lois, à un jeu de règles, à des pratiques sociales et à des concepts soumis à une sorte de dispersion. A ce titre, si nous entreprenons de saisir la formation discursive islamiste du point de vue de $\mathrm{M}$. Foucault, nous reconnaîtrons le discours comme l'un des paradigmes de cette formation discursive. Dans cette perspective, l'accent est plutôt mis sur les idées, les méthodes, les concepts et les relations - sans cadre apparent - qui détermineraient l'islamisme comme système donnant naissance à des énoncés islamistes et non comme manifestation discursive à part entière. À ce titre, M. Foucault formule l'hypothèse selon laquelle " les énoncés différents dans leur forme, dispersés dans le temps, forment un ensemble s'ils se réfèrent à un seul et même objet » (Foucault 1969 : 45) sans préciser pour autant la nature de la différence ou les limites de la dispersion. Même constat pour la démarche de M. Pêcheux qui ne pourrait saisir l'islamisme que par le biais des manifestations langagières de l'idéologie qui le gouverne par opposition à d'autres positionnements idéologiques. Dans cette logique, les deux démarches ne pourraient pas saisir le discours islamiste comme phénomène discursif, mais comme l'un des aspects systémiques, avec d'autres, composant la mouvance islamiste.

D. Maingueneau, conscient de la difficulté que suscite cette notion, a proposé un cadre plus précis afin de l'inscrire dans une problématique générale de l'analyse de discours. Pour l'analyste, la formation discursive est un espace où les unités ne sont pas limitées par les frontières posées par le chercheur et dont les énoncés sont profondément inscrits dans l'histoire. Ainsi, le corpus peut être assez hétérogène dans le sens où il pourrait mêler corpus d'archive et un corpus construit par le chercheur (test, entretien ou questionnaire) (Ibid.). Maingueneau précise que l'intérêt de la formation discursive réside dans le fait :

Qu'elle permet de constituer des corpus hétérogènes, de rassembler librement des énoncés relevant de divers types d'unités topiques. Il en résulte que la formation discursive ne recouvre pas une réalité homogène ; en fonction du critère en vertu duquel on rassemble les textes qu'on y intègre, on peut en distinguer divers types (Maingueneau 2014 : 84).

La distinction, dont il est question ici, permet d'identifier deux types de formations discursives : les formations discursives d'identité et les formations discursives thématiques. Dans le premier cas, il s'agit du postulat selon lequel les énoncés relevant d'une même formation discursive concourent vers un «foyer invisible », une pensée, qui gouvernerait discrètement les paroles (Ibid.). Cependant, il n'est pas nécessaire de partir de ce postulat-là pour mener des recherches sur une formation discursive donnée ; étant donné que cette 
dernière est foncièrement hétérogène et qu'elle peut ne pas renvoyer à un but secret. Elle s'inscrirait justement dans le cadre d'un interdiscours qui la modifierait continuellement en fonction des positions idéologiques. La seconde renvoie à une autre manière de constitution de la formation discursive, non pas sur la base des instances émettrices du discours, mais à partir d'un thème ; cela revient «à définir les énoncés qui en parlent » (Ibid. p. 87).

Prendre conscience de l'origine et de l'évolution de la notion de formation discursive est nécessaire. Cela permet de prendre en considération le volet idéologique et social qui compose le discours islamiste et de présenter un cadre à la fois précis et vaste dans lequel s'inscrivent les unités non-topiques.

\subsection{Le discours islamiste comme formation discursive dans une problématique de l'analyse de discours}

Dans le champ politique, nous sommes en mesure de saisir l'islamisme du parti islamiste selon que les énoncés se rapportent à une identité commune, malgré l'hétérogénéité des genres et des types, ou qu'ils renvoient à la thématique de l'islamisme comme mode de gestion politique. Nous sommes conscients que cela ne pourrait se faire dans un espace aussi restreint que celui d'un article ; mais nous tenterons ici de dresser un tableau sommaire, à l'aide de quelques exemples et énoncés, de ce que pourrait représenter le discours islamiste du PJD comme formation discursive.

Quand on considère les énoncés des leaders politiques islamistes, prononcés dans le cadre de genres et de types de discours, où au contraire, ceux qui sont prononcés hors de tout genre institué, nous relevons une référence claire à Sayyed Qutb et à ses principaux ouvrages FîZilâl al-qur'ân et Ma'alim fi'lTariq ${ }^{x x i}$. Dans ce sens, Abdelkrim Mouti', fondateur de la Chabiba Islamiyaxxii (la Jeunesse Islamique), s'est inspiré des idées des Frères musulmans afin de mobiliser les jeunes au sein de l'université marocaine. De même, pour Abdelkrim El-Khatib ${ }^{\text {xxiii }}$ dont le message s'approche étroitement de celui de S. Qutb, surtout lorsqu'il affirme, en 1984, lors des élections législatives, que « la crise générale que traverse le Maroc est due à l'abandon de la voie islamique par le pouvoir » (Dialmy 2000). A son tour, A. Benkirane dit, lors d'un entretien accordé au journal Média 24 du 27 novembre 2014, : " je lis un livre de Sayyed Qutb. Le lendemain, je suis une autre personne ». Même quand il prend la parole dans certains meetings, A. Benkirane précise que : « les Frères musulmans sont notre première école, celle au sein de laquelle nous avons tout appris. Nos premiers référentiels culturels sont l'école des Frères musulmans et les écrits de Hassan El Banna et Sayed Qutb. Nous leur devons beaucoup » ${ }^{x i v}$. Par ailleurs, A. Benkirane, joint par téléphone par la chaîne qatarie $A l$ Jazeera ${ }^{\mathrm{xxv}}$, a réagi à la mort du Frère musulman Mohamed Morsi : «Mohamed Morsi, dit-il, est un martyr qui s'est sacrifié pour la justice. Il n'a jamais été accusé de corruption et œuvrait chaque jour pour améliorer la situation politique et économique de son pays ». Ce dernier énoncé, dont le genre conversation téléphonique - n'est pas institué, converge vers l'idée selon laquelle le Parti de la Justice et du Développement serait en phase avec le « comportement » et l'idéologie islamiste des Frères musulmans.

Les réflexions, qui précèdent, ont permis de donner un aperçu des énoncés renvoyant à une identité commune, ou ceux qui relèvent de la thématique de l'islamisme comme mode de gestion politique. Il s'agit, à présent, d'évoquer un aspect peu commun mais significatif de cette formation discursive, eu égard à la démarche adoptée dans cette analyse. En effet, A. Benkirane, au moment où il a quitté le gouvernement ${ }^{\mathrm{xxvi}}$, n'a pas hésité à maintenir le contact avec son auditoire, constitué d'internautes et de " fidèles », en ayant recours à des vidéos diffusées sur la plateforme Youtube. L'ex-chef du gouvernement marocain est filmé, dans son domicile, entouré de ses « frères » et animant ainsi des « causeries ». Cela rappelle les méthodes d'enseignement et d'éducation politique et idéologique de feu cheikh Abdessalam Yassine, fondateur et chef spirituel du mouvement marocain : Al Adl Wal 
Ihsane (Justice et bienfaisance). Les sujets abordées par Benkirane sont polémiques : ils relèvent à la fois du fait politique et de l'importance de la religion ${ }^{\mathrm{xxvii}}$; ceci montre notamment l'usage que fait l'orateur du discours religieux comme type de discours. A travers ces vidéos, A. Benkirane conteste l'action du gouvernement El Othmani (son successeur à la tête du gouvernement et du parti) et remet en cause certaines de ses décisions et de ses actions. Sur une vidéo diffusée le 20 juillet 2019, le montrant dans son salon à son domicile de Rabat, l'ex-chef du gouvernement incitait les députés du PJD à ne pas voter le projet de loi-cadre sur l'enseignement et d'aller contre les directives d'El Othmani ${ }^{\text {xviii }}$. Certes, Benkirane s'exprime en qualité de principal fondateur et leader mais le cadre scénique n'est pas représentatif d'un lieu institutionnel qui permet aux internautes d'identifier le discours en tant que message politique.

Ainsi, le message du sujet communiquant ne peut être légitimé par le destinataire interprétant. C'est pourquoi, nous ne pouvons pas en général placer le «live Youtube », tel qu'il est conçu par A. Benkirane, dans la catégorie des genres institués. Ceci dit, le message de Benkirane s'inscrit dans une entité qui subsume les genres institués formant ainsi la formation discursive islamiste.

Nous ne pouvons pas parler ici d'une formation discursive au sens complet du terme. Néanmoins, cette description permet de dégager les relations idéologiques qui surgissent à travers les énoncés des uns et des autres. Cette esquisse permet de tracer quelques aspects de ce que représenterait une formation discursive islamiste et ses liens profonds. Le rapport intitulé « La Fabrique de l'islamisme », publié par l'Institut Montaigne en 2018, souligne ce lien étroit qui existe entre les deux tendances. Les discours, énoncés çà et là par les cadres du PJD, convergent vers les principes fondateurs de l'idéologie des Frères musulmans ; idéologie qui soutient l'idée selon laquelle la société est en état de dépravation du fait qu'elle n'applique plus les préceptes de l'islam et défend la nécessité de la réislamisation de la société par le Jihad (la guerre sainte).

\section{Conclusion}

Nous avons tenté, à travers ce travail, de tracer quelques contours de l'islamisme du Parti marocain de la Justice et du Développement comme activité discursive, et ce à travers les unités topiques et la notion de formation discursive. En effet, l'analyse des unités domaniales montre que la sphère d'activité est caractérisée par une scénographie qui ne se modifie pas beaucoup. Les lieux d'activité sont assez classiques. Cependant, l'activité discursive est prégnante sur Internet et les réseaux sociaux. Internet permet en effet la circulation rapide des contenus, et les réseaux sociaux une diffusion extrêmement large. L'outil numérique est devenu le canal de diffusion pour le discours du PJD. On note notamment les références multiples à la confrérie des Frères musulmans de l'Égypte et surtout aux textes de leur principale idéologue, Sayyed Qutb.

L'expression discursive du PJD se manifeste aussi à travers les références et les oppositions qu'elle entretient avec l'association $\mathrm{Al}$ Adl $\mathrm{Wa}$ Al Ihssan (Justice et bienfaisance) de Cheikh Yassine. Le discours islamiste du PJD, se manifeste à travers des unités domaniales, autour de l'activité politique marocaine, et à travers une formation discursive dont les unités montrent un attachement souterrain à la confrérie mère, celle des Frères musulmans d'Égypte.

Il faut certes élargir notre analyse et explorer l'islamisme marocain, de manière à étudier le réseau de relations entretenues entre les formations islamistes au Maroc, dégager leurs oppositions et mieux identifier la nature des liens entretenus avec les Frères musulmans égyptiens, notamment à travers les parcours. 


\section{Références bibliographiques}

Adam, J.-M. (1997). « Unités rédactionnelles et genres discursifs : cadre général pour une approche de la presse écrite », Pratiques, n 94 , p. 3-18.

Belal, Y. (2009). «L'usure des oppositions islamistes au Maroc », L'Année du Maghreb, V, pp. 5974.

Benveniste, E. (1966). Problèmes de linguistique générale, Paris : Gallimard.

Carré, O. (1984). Mystique et politique. Lecture révolutionnaire du Coran par Sayyid Qutb, Frère musulman radical. Paris : Presses de Sciences Po.

Charaudeau, P. (1983). Langage et discours. Éléments de sémiolinguistique. Paris : Hachette.

Dialmy, A. (2000). «L'islamisme marocain : entre révolution et intégration », Archives de sciences sociales des religions, $\mathrm{n}^{\circ} 110$, pp. 5-27.

Foucault, M. (1969). L'Archéologie du savoir. Paris : Gallimard.

Haroche, C., Henry, P., Pêcheux, M. (1971). « La sémantique et la coupure saussurienne : langue, langage, discours ». Langages, ${ }^{\circ} 24$, pp. 93-106.

Kerbrat-Orecchioni, C. (1992). Les interactions verbales. Paris : Armand Colin.

Larroque, A.-C.(2018). L'islamisme au pouvoir : Tunisie, Égypte, Maroc. Paris : P.U.F.

Le Bart, C. (1998). Le discours politique. Paris : P.U.F.

Maingueneau, D. (2005). «L'analyse du discours et ses frontières ». Marges linguistiques, $\mathrm{n}^{\circ}$, pp. 64-75.

Maingueneau, D. (2009). Les termes clés de l'analyse du discours. Paris : Seuil.

Maingueneau, D. (2010). « Le discours politique et son environnement ». Mots. Les langages $d u$ politique, $\mathrm{n}^{\circ} 94$, pp. 85-90.

Maingueneau, D. (2011). « Pertinence de la notion de formation discursive en analyse de discours ». Langage et société, $\mathrm{n}^{\circ} 135$, pp. 87-99.

Maingueneau, D. (2012). Analyser les textes de communication. Paris : Armand Colin.

Maingueneau, D. (2014). Discours et analyse de discours. Paris : Armand Colin.

Mejdoubi, S. (2013). Isotopie et métaphore dans le discours politique islamiste au Maroc : Cas du Parti de la Justice et du Développement, Thèse de doctorat, Rabat, Université Mohamed V.

Tozy, M. (1984). Champ et contrechamp politico-religieux au Maroc, Thèse de doctorat, Marseille, Université Aix-Marseille.

Ziamari, K., De Ruiter, J.-J. (2015). « Les langues au Maroc : réalités, changements et évolutions linguistique ». Le Maroc au présent : d'une époque à l'autre, une société en mutation. Casablanca, Centre Jacques-Berque, pp. 441-462.

\footnotetext{
'Avant 2011, date à laquelle le Maroc a connu un bouleversement politique, le Parti de la Justice et du Développement n'a jamais été au pouvoir et l'état contrôlait ses manifestations et son discours. Depuis, l'horizon de la parole du PJD s'est élargie.

ii Les langues, en œuvre au Maroc, et leurs usages représentent un cas assez intéressant qui mérite que l'on s'y attarde. D'une manière sommaire, nous pouvons expliquer, si l'on écarte l'amazigh et les parlers régionaux, que lorsqu'il s'agit d'échanges langagiers ou de
} 
discours politiques dans un cadre institutionnel, deux langues sont en confrontation : l'arabe standard et le parler marocain. Cette dualité est au centre des débats et des polémiques qui ont animés les scènes politique et médiatique au Maroc et la constitution de 2011 n'a pas apaisé les esprits. Alors que les constitutions précédentes, qu'a connues le Maroc depuis son indépendance en 1956, n'ont reconnu que la langue arabe comme l'unique langue officielle du royaume, celle de 2011 élargit timidement sa reconnaissance à la langue amazighe comme " une " langue officielle car elle relevait du patrimoine commun aux marocains. Cette constitution mentionne, entre autres, qu'elle reconnaît les parlers pratiqués et encourage l'apprentissage des langues étrangères. Malgré ce positionnement quant à la diversité linguistique, la constitution de 2011 ne mentionne pas explicitement la place qu'occupe le parler marocain en tant que langue maternelle et persiste à reconnaître une langue officielle qui n'est pas parlée et vilipende une langue légitime largement utilisée par les locuteurs marocains. Le marocain est la langue qui permet l'expression des sentiments, des besoins et à travers laquelle s'effectue la saisie de l'ensemble des univers auxquels s'identifient les marocains.

iii D. Maingueneau identifie différentes lois qui gouvernent le discours dans son ouvrage Analyser les textes de communication, pp. 18-28.

iv A ce titre, nous renvoyons le lecteur aux multiples sorties médiatiques de Benkirane et de El Othmani, dont les extraits sont largement relayés par les médias sociaux.

$\checkmark$ Un espace discursif est un espace plus limité à l'intérieur d'un sous-champ défini.

vi Maroc diplomatique, 4 février 2019 : https://maroc-diplomatique.net/ramid-etmaelainine-declarent-la-guerre-des-mots/

vii Ces genres sont à retrouver en langue arabe sur le site du parti : https://www.pjd.ma/epace-institutionnel/الحزب

viii le le contenu en arabe :

https://www.pjd.ma/sites/default/files/communiques/lhwr Idkhlykhlst wtwsyt 2019.pdf

ix Ces références au parlement, énoncées en arabe ou en parler marocain, lors des interventions des ministres et des chefs de gouvernement du PJD, ne peuvent être entendues qu'à travers la chaine de télévision qui transmet directement les séances hebdomadaires, ou alors à travers quelques vidéos que l'on retrouve sur la plateforme Youtube.

× Deux ans après les législatives de 2007 et un an après avoir été rebaptisé : Parti de la Justice et du Développement.

${ }^{x i}$ Que l'on peut traduire par "le renouveau ».

xii Association fondée en 1996 par Ahmed Raïssouni et Abdelilah Benkirane et dont le principal objectif est la prédication religieuse.

xiii Tel que Mustapha El Khalfi, éditorialiste, rédacteur en chef et directeur de la publication de 2007 à 2012. A partir de 2012, il est nommé ministre de la communication sous le gouvernement Benkirane et actuel ministre délégué auprès du chef du gouvernement, El Othmani, chargé des Relations avec le Parlement et la société civile, porte-parole du gouvernement.

xivhttps://www.leseco.ma/medias/82764-le-pjd-lance-son-magazine.html

${ }^{x v}$ Rassemblant plusieurs formations politiques afin de débattre des réalisations et des projets du gouvernement.

xvihttps://www.pjd.ma

xviihttp://radio.pid.ma

xviiihttp://tv.pjd.ma 


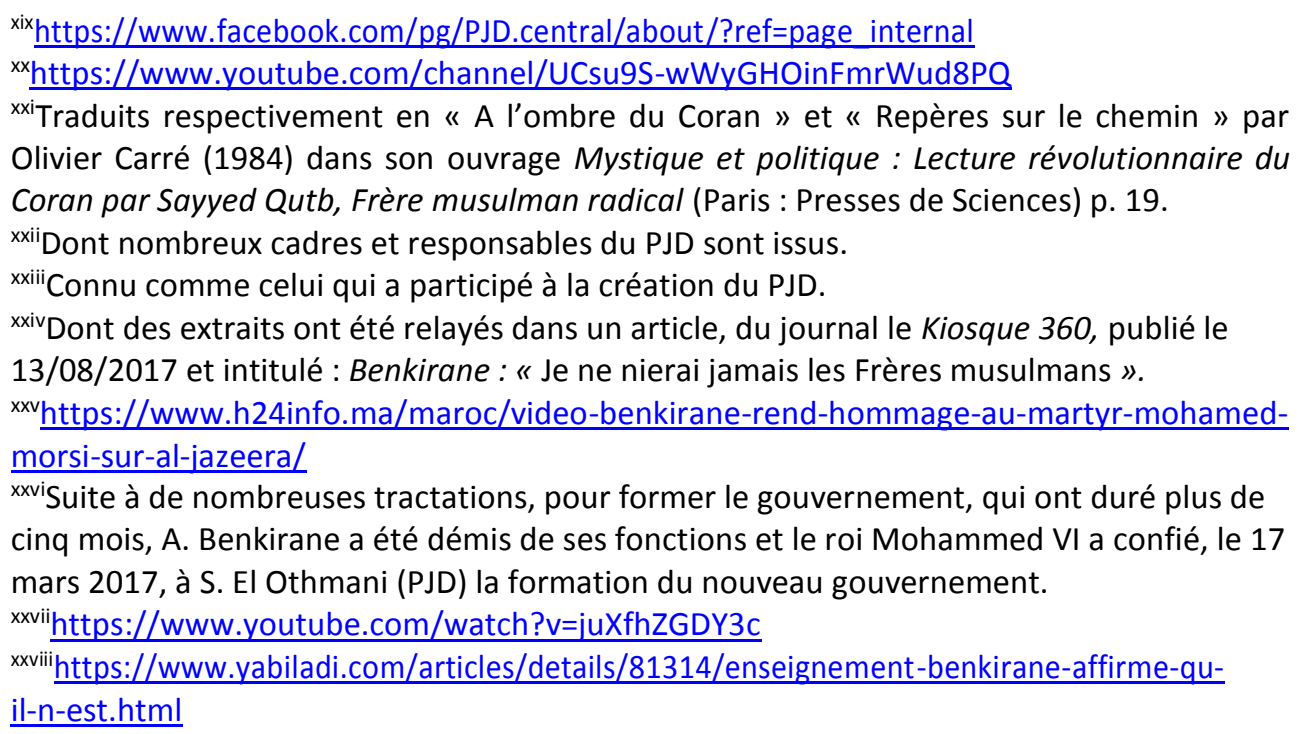

